\title{
Adaptation of Web Services Based on QoS Satisfaction
}

\author{
Barbara Pernici and S. Hossein Siadat \\ Politecnico di Milano, Italy \\ \{pernici, siadat\}@elet.polimi.it
}

\begin{abstract}
Requirements of Service Based Applications (SBAs) tend to change during the life cycle of the service. Therefore, adaptation and evolution of services become a necessity in order to provide the agreed Quality of Service (QoS) stated in a contract between the service provider and requestor. Recently, many adaptation methods have been proposed in the literature. However, there is no overall consensus in selecting the best strategy and the consequences of adaptation are usually neglected. In this paper, we propose an approach for service adaptation through defining a flexible service description using fuzzy parameters. This approach provides a compatibility mechanism that measures the aggregated satisfaction value of offered services to understand to what extent the quality changes are satisfiable according to the existing contract. According to the degree of satisfaction function we then propose our adaptation/evolution strategy.
\end{abstract}

\section{Introduction}

With the rapid propagation of Service Based Applications (SBAs), evolutionary changes of Web Services are gaining huge interest among the practitioners. Changes are due to several reasons and may occur continuously over time. Such changes need to be captured and analyzed as they have various requirements, causes and effects. These changes then will be compared with Service Level Agreement (SLA) which is a guarantee of a certain agreement between the service provider and the requestor of the service. This paper is concerned with non-functional requirements of services particularly QoS issues, and we investigate QoS changes through a contractual approach. Therefore, we define a fault/violation when a service is not delivered according to the predefined quality description in the contract. Typical non-functional properties include availability, response time, cost and throughput are often collectively referred to as quality dimensions. While 18, provides a general categorization of SLA metrics, 24, 21] and [19] present a description of QoS attributes and frameworks for Web service selection and discovery. It is possible to define QoS description as an XML specification of SLAs in several languages such as Web Service Level Agreement (WSLA) [10] by IBM or Web Service Management Language (WSML) [20] by HP and WS-Agreement [3]. 
Deviation of quality ranges from the existing contract may produce a system failure and bring dissatisfaction for customers. To this end, the evolution and adaptation of web services are becoming two important issues in reacting to the various changes in order to provide the agreed QoS stated in the contract. Recently, many adaptation strategies and methods have been proposed in the literature. A list of adaptation strategies for repair processes in SBAs is provided in [6] and [1].

However, there is no overall consensus in defining and selecting the best strategy when a change occurs. Furthermore, business aspects like cost, the consequences of adaptation actions, say service replacement, and customer satisfaction are usually neglected. This is of great importance to consider the cost of selecting another service provider and establish a new contract which could be time consuming as well in some circumstances. Taking into account that, in many cases selecting a new service provider as an adaptation action is a costly decision without significant achievement with respect to QoS. Therefore, any service replacement needs to be analyzed specially when there is a high degree of loyalty between the involving parties in long term contracts.

We address these limitations by defining a service satisfaction degree that allows us to select an appropriate adaptation action. The research challenge we are following in the paper is twofold. Firstly, how to provide compatibility between the provider and the requestor according to the existing contract once the QoS is changed? Second, how to define a mechanism for selecting an appropriate adaptation strategy. In order to address these research challenges a few steps are required.

1. Defining the service description in a flexible approach to describe quality of the provided service. For this, we extend the service description defined in [4 by using a fuzzy approach for defining quality dimensions. This way we understand to what extent a quality parameter is satisfied/violated. Quality parameters are usually defined in a strict and non-flexible manner. Even when they were defined within ranges, any value out of the range is considered to be a violation. Therefore, a monitoring engine behaves in a binary approach which results in either violation or not. In this paper, we consider a fuzzy approach for measuring violation of each parameter. Therefore, we are able to measure the degree of violation/satisfaction of each quality parameter.

2. Providing a satisfaction function taking advantages of the service description equipped with fuzzy parameters. According to the satisfaction degree gained from the satisfaction we then propose an adaptation strategy to be performed. In the following, we start by a motivating example in Section 2 . Section 3 introduces the major related work. In Section 4 we show the formalization of service description through defining fuzzy parameters and in Section 5 we define a satisfaction function to measure to what extend the QoS is achieved according to the existing contract. We then define the extended compatibility mechanism and adaptation algorithm in Section [6 that work based on the result of the satisfaction function. Adaptation strategies will be discussed in Section 7 and we conclude the paper in Section 8. 


\section{Motivating Example}

Here we provide a motivating example to illustrate the service adaptation problem. Suppose that there is an airline agency providing plane tickets for its customers. It provides different services offered by various airlines. Consider that each service is associated with three parameters of Availability, Response Time and Cost (other aspects i.e. flight time and number of stopovers are applicable). Customers show a rather relaxed behavior for the availability and response time of the services, but they are very strict with the Cost. Underlying this example there is the following assumption: Customers have strong commitment for their provider, therefore they are interested in using services from the same provider.

Suppose that a service is found and a contract is negotiated between a provider and a customer such that it offers a flight from location A to B with defined quality parameters. The quality of the offered service can dynamically change during the execution. For example, the service provider has a delay in providing the offered service. In such cases, an adaptation strategy needs to be performed in order to comply the violated situation. We consider two main levels of adaptation actions. The first and the easiest one is to choose another service provider that offers a service with the same functionality (i.e. providing a flight from location A to B), however the new service probably has different QoS guarantees. This approach is interpreted as service replacement. The second option is to renegotiate internally between the same provider and customer. For example the violation could be compensated by offering a lower price for the service. Before taking any action, some criteria need to be taken into account and from different perspectives. A new service provider may offer a service with a better quality, however the cost and consequences of choosing another provider need to be evaluated. This includes the cost of extra queries need to be done to find the best new provider and also the cost of a new contract that should be established. There are other issues involved from the customer perspective, for example he may have preferences to remain with the current provider due to the loyalty aspects. However, the internal renegotiation may not be possible all the time if the violation level leads to the dissatisfaction of customers (i.e. if the offered service exceeds the maximum budget). Considering all the aforementioned points, taking an option with best quality offered is not the ideal solution. There is a need to trade-off between the overall value of the offered quality and the cost factor. Fig. 1 shows an overall model of the approach.

\section{Related Work}

Recently, service adaptation and evolution in SBAs have gained increasing attention among the practitioners and researchers. Chafle et al. 7] presented A-WCSE approach for adaptive web service composition and execution. The approach allows adaptation by generating multiple workflows at different stages. Canfora et al. 5] presented an approach for QoS-aware replanning of service composition at run time using late-binding technique. As a result there is much previous work 


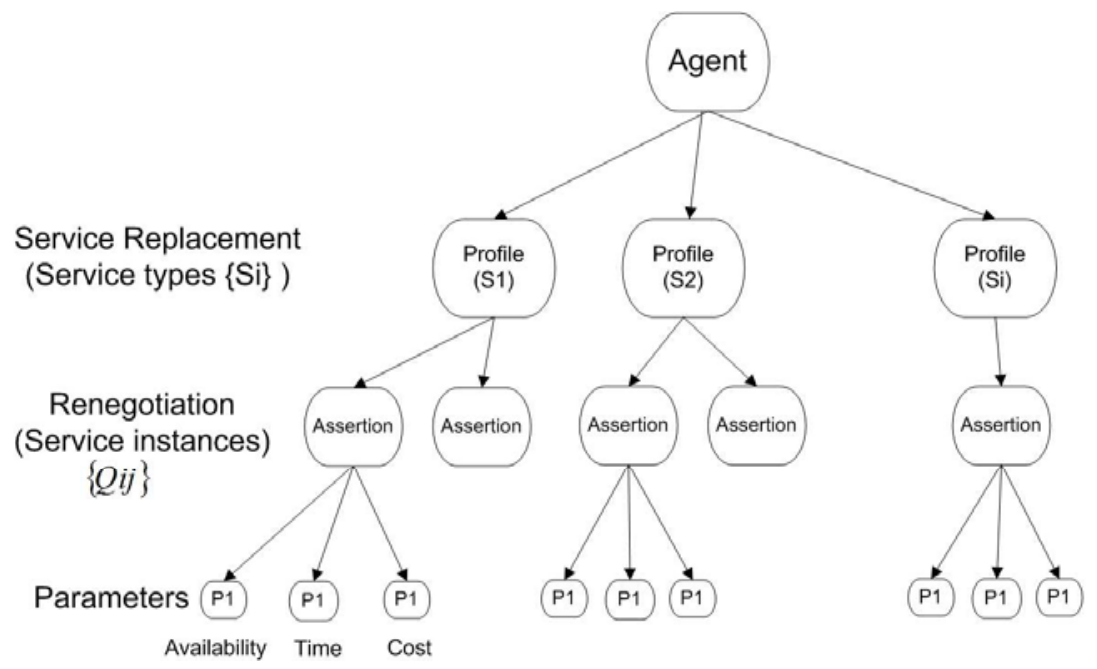

Fig. 1. Motivating Example (Overall approach)

on adaptation strategies and techniques. However, there is no overall consensus in selecting the best strategy and the consequences of such adaptation strategies are usually neglected. For this purpose some research has been investigated to consider the overall value of a change. Harney and Doshi [8] presented a mechanism called Value of changed information (VOC) to estimate the overall value brought by a change. He et al. 9] has applied the VOC mechanism for the adaptation of web service based on workflow patterns. None of the above approaches consider a satisfaction level for the offered service.

We argue that the problem lies in defining a flexible description for Web Services. Although a lot of research has been conducted for functional Web Service description, only a few efforts have been done with respect to non-functional properties description of Web Service. Among syntactic and semantic WS description we refer to the works done by [15] and [11] which they also provided algorithms for service selection based on such description. A major limitation of these works and other similar ones is due to not considering the partial satisfaction of the QoS attributes. With this regards, [17] provided a semantic Policy Centered Meta-model (PCM) approach for non-functional property description of web services. A number of operators (e.g. greaterEqual, atLeast) for numeric values are defined in the model for determining tradeoffs between various requests. Therefore, the approach can support the selection of Web Services that partially satisfy user constraints. In [13] and [12], the authors extend the approach proposed in [17] by proving a solution for Web Service evaluation based on constraint satisfaction problem. The approach uses utility function to present the level of preferences for each value ranges defined in the service description. However, is does not take care of adaptation issues and controlling values at run-time. In [16], the authors discuss about fixable and non-fixable properties 
to deal with bounded uncertainty issue. Constraint programming is used as a solution for the said issue, however, there is no evaluation of the work.

Fuzzy approach 23 has been used in several systems and application to perform the fuzzy behavior. [14 defined a set of fuzzy requirements in software development. We used fuzzy parameters in defining service description to evaluate the satisfaction degree of each parameter and to perform an appropriate adaptation action based on the satisfaction level.

\section{Fuzzy Parameters for Quality Dimensions}

This section is devoted to demonstrate the formal definitions of parameters, profiles and assertions in a service description. The formal specification we propose has been inspired and is an extension of our previous work 4]. We extended the work by taking advantage of fuzzy approach providing two kinds of parameters namely fuzzy and non-fuzzy. Having introduced the fuzzy parameters it is possible to understand to what extent the quality parameters are violated/satisfied.

In order to formally define fuzzy parameters, we need to introduce some background from [4. We define set $\mathcal{D}$ to contain the quality dimensions (such as availability, execution time, price or throughput) identified and agreed by the service provider and consumer. Each quality dimension has a domain and range; e.g., availability is a probability usually expressed as a percentage in the range $0-100 \%$ and execution time is in the domain of real numbers in the range $0 . .+\infty$. A quality dimension $d$ can be considered monotonic (denoted by $d^{+}$) or antitonic $\left(d^{-}\right)$; monotonicity indicates that values closer to the upper bound of the range are considered "better", whilst with antitonic dimensions values closer to the lower bound are considered better. A parameter $m$ associates a quality dimension to a value range.

If a parameter is non-fuzzy (strict), its satisfaction degree will be evaluated in a binary manner (which is a Yes or No). In contrast, fuzzy parameters (relaxed) will be evaluated in a fuzzy manner which shows different degree of satisfaction $(x \in[0,1])$. Note that we also provide value ranges for both parameters regardless of being fuzzy or non-fuzzy. The degree of satisfaction will be evaluated using a satisfaction function we introduce in the next section. In the following we provide the extended definition of a parameter based on the definition introduced in 4 .

Definition 1 (Parameter). We define a Parameter $m \in \mathcal{M}$ as a tuple $m:=$ $(d, v, f), d \in \mathcal{D}, v \in \mathcal{V}, f \in\{s, r\}$. where $\mathcal{D}$ is the set of quality dimensions, $\mathcal{V}$ is the set of ranges for all quality dimensions $\mathcal{D}$, s represent a strict parameter and $r$ represent a relaxed parameter.

Having defined the quality dimensions and service description, a contract will be defined based on a matching and mapping functions defined in our previous work [4] in a similar manner. We do not present the functions here as they are not the concern of this paper.

The definition of assertion is the same as in 4. Assertions can be combined in a similar fashion to form a profile. For the sake of simplicity we also skip the definitions of assertion and profile. 
Table 1. Working example: formal specifications

\begin{tabular}{|c|c|}
\hline \multirow{3}{*}{ Provider $\mathcal{P}$} & $l_{p}=\left(q_{p 1}, q_{p 2}\right)$ \\
\hline & $\begin{array}{l}q_{p 1}=\left(m_{p 1,1}, m_{p 2,1}, \overline{m_{p 3,1}}\right) \\
m_{p 1,1}=\left(d_{1},[0.8,0.95]\right), m_{p 2,1}=\left(d_{2},[2,5]\right), \overline{m_{p 3,1}}=\left(d_{3},[1,1]\right)\end{array}$ \\
\hline & $\begin{array}{l}q_{p 2}=\left(m_{p 1,2}, m_{p 2,2}, \overline{m_{p 3,2}}\right) \\
m_{p 1,2}=\left(d_{1},[0.7,0.95]\right), m_{p 2,2}=\left(d_{2},[3,5]\right), \overline{m_{p 3,2}}=\left(d_{3},[0.8,0.8]\right)\end{array}$ \\
\hline \multirow[t]{2}{*}{ Consumer $\mathcal{C}$} & $l_{c}=\left(q_{c 1}\right)$ \\
\hline & $\begin{array}{l}q_{c 1}=\left(\overline{m_{c 1,1}}, \overline{m_{c 2,1}}, m_{c 3,1}\right) \\
\overline{m_{c 1,1}}=\left(d_{1},[0.8,0.9]\right), \overline{m_{c 2,1}}=\left(d_{2},[3,5]\right), m_{c 3,1}=\left(d_{3},[0,1]\right)\end{array}$ \\
\hline Dimensions $\mathcal{D}$ & $d_{1}^{+}=$Availability, $d_{2}^{-}=$ResponseTime, $d_{3}^{-}=$Price \\
\hline
\end{tabular}

During a service life-cycle, as the application runs, QoS offerings may change due to several reasons. Therefore, adaptation of web services needs to be performed in an appropriate manner to accommodate QoS changes/violations by choosing the best adaptation strategy. Defining service description with the aforementioned fuzzy parameters provides a more flexible situation dealing with adaptation decisions. We discuss how it can facilitate the adaptation of web services through our motivating example according to Table1. According to the new definition of parameters we already explained, let us assume that Availability and ResponseTime are fuzzy parameters and Cost is a non-fuzzy Parameter.

In 4 we provided situations in which new QoS ranges could be still acceptable for both parties according to the existing contract. We defined a compatibility mechanism that uses parameter subtyping and used Allen's Interval Algebra [2] in order to express the subtyping. The provider and requestor are compatible with each other according to the existing contract if the QoS changes are in one of the acceptable situations. If the compatibility is not provided, however it does not give any information about the degree of satisfaction/dissatisfaction of the offered service. Let us consider an availability of 80 to $90 \%$. For example if the new range of availability is less than $80 \%$, this is not considered as an acceptable situation and it is considered as a violation. In such cases, we would also like to understand to what extent the quality parameter and the aggregated service quality are satisfactory. An availability of $75 \%$ might still be acceptable if we consider a general value of all parameters. In the following we will concentrate on the definition of a more precise compatibility mechanism taking advantage of the fuzzy approach we have already presented. To this end, we introduce a satisfaction function in the next section.

\section{Satisfaction Function}

We associate a satisfaction value of $S \in[0,1]$ to each parameter. In order to calculate the satisfaction value $A(m)$ for a given parameter $m$, we use Equations 1 and 2 for monotonic and antitonic parameters respectively as follow:

$$
A(m)= \begin{cases}m^{\prime} \div m_{\text {min }} & \text { InCompatible } \\ 1 & \text { Compatible }\end{cases}
$$


Note: Equation 1 and 2 are only applicable for fuzzy parameters while non-fuzzy parameters receive a satisfaction value of 0 in case of incompatibility.

For monotonic dimensions: the range between 0 and the minimum range in the contract will be mapped to $[0,1]$. This is actually the area of violation according to the contract (reported as negative weight in some work). Any value between the min and max is considered to be compatible (satisfaction value of 1 ). For antitonic dimensions, the range between 0 and maximum value in the contract is considered to be compatible (the satisfaction value 1) and the range between maximum value and the actual new value is mapped to the satisfaction value of $[0,1]$.

$$
A(m)= \begin{cases}m_{\max } \div m^{\prime} & \text { InCompatible } \\ 1 & \text { Compatible }\end{cases}
$$

In order to calculate the overall satisfaction degree of the service including satisfaction value of all the parameters, we can apply the following method in Equation 3. Consider $A(Q)$ as the satisfaction of the assertion $Q$ that has a set of parameters. Since the parameters are structured in an AND-refined approach with $s$ strict and $f$ fuzzy parameters, therefore, the overall satisfaction value could be measured as below:

$$
A(Q)=(A(M i) \wedge \ldots \wedge A(M s)) * \min (A(M j), \ldots A(M f))
$$

$A(M i)=0,1 \quad i=1, \ldots, s$ (satisfaction value of strict parameters) $A(M j) \in[0,1] \quad j=1, \ldots, f$ (satisfaction value of fuzzy parameters)

It can be interpreted from Equation 3 that the overall satisfaction of the service depends on the minimum satisfaction of its fuzzy parameters. The offered QoS will not be satisfied if at least one of the strict parameters is not satisfied. For example if the cost is more than the maximum amount, the overall satisfaction value is 0 . As for the fuzzy parameters, let us assume that the perceived availability is $70 \%$ (the range in the contract is between $80 \%$ to $90 \%$ ), and the response time is $3 \mathrm{~s}$ (compatible according to range in the contract). Therefore, according to Equation 1 the satisfaction value is 0.88 and according to Equation 3 the overall service satisfaction is 0.88 . Now, let us consider the case that the response time is $6 \mathrm{~s}$, then according to the Equation 2 the satisfaction value of time parameter is 0.83 and therefore, the overall service satisfaction is 0.83 .

The satisfaction value could be compared with the satisfaction value of other assertions of the current web service or with the value of other web services in the composition. The existing contract will remain valid between the service provider and the customer as long as the satisfaction value is more than any other services in the composition. While the contract is not valid when the satisfaction value is less than an agreed value or a service provider with a better value is found. A service replacement may occur in this situation. Besides from the satisfaction factor, we introduce a loyalty degree which is a degree of commitment between a provider and consumer. The service loyalty/fidelity degree is taken into account before any decision for adaptation. This is due to the fact that both customer and service provider (involving parties) want to remain with the existing contract 
(specially for the long term contracts). We therefore define a total satisfaction degree using the loyalty degree as below in Equation 4 .

$$
A_{t}(Q)=A(Q) * \text { LoyaltyDegree }
$$

Let us consider a loyalty degree of 1.1 between the customer and service provider, therefore, the overall satisfaction value of the previous example will be 0.91 $\left(0.83^{*} 1.1\right)$. An initial degree of loyalty could be given to a provider and specified in the contract by the customer. In the next section we provide an algorithm for dealing with adaptation/evolution decisions.

\section{Compatibility Mechanism}

This chapter presents an algorithm for choosing the best adaptation/evolution strategy once a QoS violation occurs. The algorithm is based on the mechanism presented in Section 5 and the compatibility mechanism we introduced in [4]. Fig. 2 shows the pseudo code for selecting adaptation actions based on the QoS satisfaction value of the offered service.

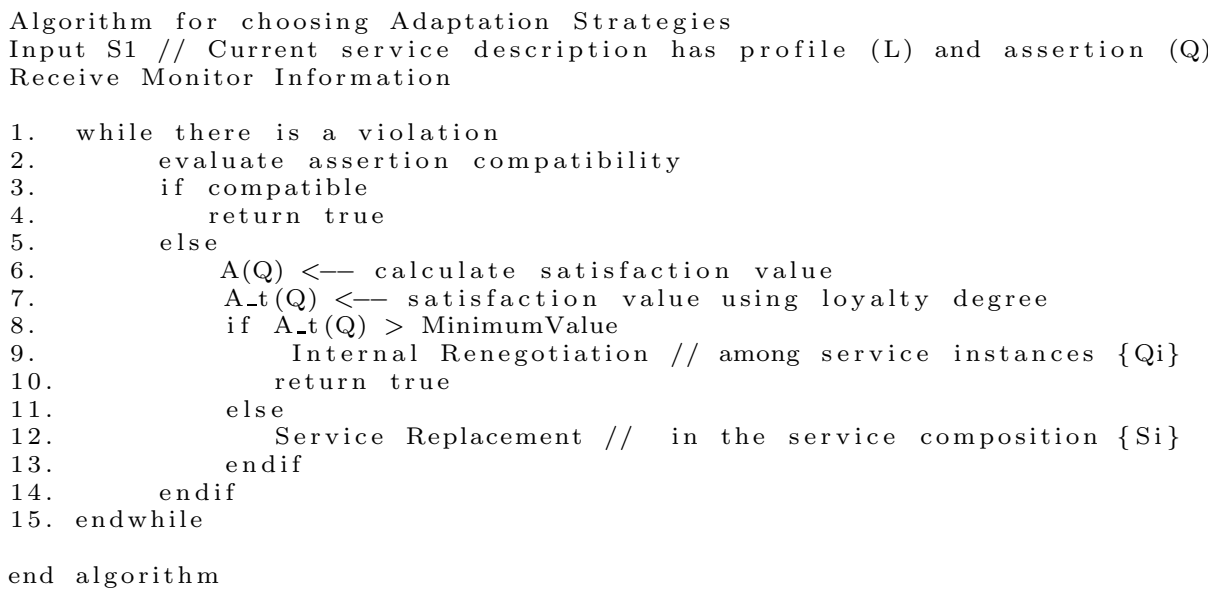

Fig. 2. Pseudo code for adaptation strategies

The algorithm takes one input that is the description of the service defined in the contract. The algorithm starts when it receives information from monitoring engine or when one party requests a new requirement. Assertion compatibility is evaluated in line 2 to identify whether the new change is according to the existing contract based on the mechanism we introduced in [4. If the change is not compatible it means that it is not within one of the acceptable situations then basically the satisfaction value is calculated (line 6 and 7) to express to what extent the contract is violated (degree of satisfaction). In line 8, the satisfaction value will be compared with a threshold (MinimumValue) that is defined in the contract. According to the degree of satisfaction, an adaptation action will be 
chosen to either renegotiate the existing contract with the same service provider (line 9) or perform a service replacement to select a new service provider and set a new contract (line 12). As it is demonstrated in Fig. 1, the internal renegotiation is at the level of assertions and among service instances while service replacement is at the level of profiles between different service types.

\section{Adaptation}

Having identified the satisfaction value and the compatibility mechanism it is possible to trigger adaptation/evolution strategies. According to the degree of satisfaction we basically propose three different categories, namely Compatible, Soft Adaptation and Hard Adaptation to perform adaptation and evolution as explained in the following:

1. Compatible: This is the situation that the involving parties are considered to be compatible with each other according to the existing contract. For this, we provided in 4 the acceptable conditions in which quality changes can take place without the need for involving parties to change. Therefore, the adaptation action is indeed Do Nothing. This adaptation is applicable for shallow changes and as a result the existing contract will not be modified. The main research challenge in this category is how to provide the compatibility in case the QoS changes.

2. Soft Adaptation: The second adaptation category is considered as an internal renegotiation between the involving parties over the existing contract. This happens when changes are not in one of the acceptable compatible conditions, therefore, the contract is not respected to some extent. According to the degree of satisfaction, this situation could be internally renegotiated so that involving parties could continue with a modified version of the contract (Penalties are applicable in some circumstances). Possible adaptation strategies are relaxing a constraint or goal, reconciliation, compensation, changing value ranges of parameters and so on. Depending on the service description it is possible to use multiple value ranges for the same quality dimension. Therefore, another action would be using an alternative assertion (our approach) or using an OR-refinement of goals in goal oriented approaches [22. Overall, changes in this category are still considered to be shallow changes however they require a contract modification which is internally and between the involving parties.

3. Hard Adaptation: The third adaptation category takes place when the contract is not valid any longer between the involving parties and there is a high degree of dissatisfaction. Possible strategies in this category include new binding, service selection or re-composition. In these approaches, the cost of service replacement, reconfiguration of the composition and setting a new contract need to be taken into the consideration. Changes are in the deep category of changes and the result of change will propagate in the entire value chain of the service composition. 


\section{Conclusions and Future Work}

This paper concerns the issue of adaptation strategies from the QoS perspective. In SBAs adaptation and evolution of services are necessary due to the various changes, however, there is no overall consensus in selecting the best strategy and the consequences of adaptation are usually neglected. In this paper, we addressed such problematic issues in service adaptation and evolution. In particular, we proposed a flexible approach to deal with QoS parameters formulated within given value ranges in a contract. We applied a fuzzy approach to define quality parameters so that their degree of satisfaction could be estimated. For that purpose we introduced a satisfaction function. Specifically, we propose an algorithm for adaptation strategies that works based on the satisfaction levels.

As part of our future work, we will implement a prototype of the proposed approach. We will also investigate to improve the satisfaction function in order to show a more realistic behavior specially for the Non-fuzzy parameters.

\section{Acknowledgements}

The research leading to these results has received funding from the European Community's Seventh Framework Programme FP7/2007-2013 under grant agreement 215483 (S-Cube).

\section{References}

1. Di Nitto, E., Kazhamiakin, R., Mazza, V., Bucchiarone, A., Cappiello, C., Pistore, M.: Design for adaptation of service-based applications: Main issues and requirements. In: The Fifth International Workshop on Engineering Service-Oriented Applications: Supporting Software Service Development Lifecycles, WESOA (2009)

2. Allen, J.F.: Maintaining Knowledge about Temporal Intervals. Communications of the ACM 26(11), 832-843 (1983)

3. Andrieux, A., et al.: Web Services Agreement Specification (WS-Agreement). Recommended standard, Open Grid Forum (March 2007)

4. Andrikopoulos, V., et al.: QoS Contract Formation and Evolution. In: Buccafurri, F., Semeraro, G. (eds.) EC-Web 2010. LNBIP, vol. 61, pp. 290-304. Springer, Heidelberg (2010)

5. Canfora, G., Di Penta, M., Esposito, R., Villani, M.L.: Qos-aware replanning of composite web services. In: ICWS, pp. 121-129 (2005)

6. Cappiello, C., Pernici, B.: Quality-aware design of repairable processes. In: The 13th International Conference on Information Quality (ICIQ 2008), pp. 382-396 (2008)

7. Chafle, G., Dasgupta, K., Kumar, A., Mittal, S., Srivastava, B.: Adaptation inweb service composition and execution. In: IEEE International Conference on Web Services, pp. 549-557 (2006)

8. Harney, J., Doshi, P.: Adaptive web processes using value of changed information. In: Dan, A., Lamersdorf, W. (eds.) ICSOC 2006. LNCS, vol. 4294, pp. 179-190. Springer, Heidelberg (2006) 
9. He, Q., Yan, J., Jin, H., Yang, Y.: Adaptation of web service composition based on workflow patterns. In: Bouguettaya, A., Krueger, I., Margaria, T. (eds.) ICSOC 2008. LNCS, vol. 5364, pp. 22-37. Springer, Heidelberg (2008)

10. Keller, A., Ludwig, H.: The wsla framework: Specifying and monitoring service level agreements for web services. J. Network Syst. Manage. 11(1) (2003)

11. Kritikos, K., Plexousakis, D.: Semantic qos-based web service discovery algorithms. In: ECOWS, pp. 181-190 (2007)

12. Li, P., Comerio, M., Maurino, A., De Paoli, F.: Advanced non-functional property evaluation of web services. In: Proceedings of the 2009 Seventh IEEE European Conference on Web Services, ECOWS 2009, Washington, DC, USA, pp. 27-36. IEEE Computer Society, Los Alamitos (2009)

13. Li, P., Comerio, M., Maurino, A., De Paoli, F.: An approach to non-functional property evaluation of web services. In: Proceedings of the 2009 IEEE International Conference on Web Services, ICWS 2009, Washington, DC, USA, pp. 1004-1005. IEEE Computer Society, Los Alamitos (2009)

14. Liu, X.F.: Fuzzy requirements. IEEE Potentials 17(2), 24-26 (1998)

15. Martín-Díaz, O., Ruiz-Cortés, A., Benavides, D., Durán, A., Toro, M.: A qualityaware approach to web services procurement. In: Benatallah, B., Shan, M.-C. (eds.) TES 2003. LNCS, vol. 2819, pp. 42-53. Springer, Heidelberg (2003)

16. Martín-Díaz, O., Ruiz-Cortés, A., García, J.M., Toro, M.: Dealing with fixable and non-fixable properties in service matchmaking. In: Dan, A., Gittler, F., Toumani, F. (eds.) ICSOC/ServiceWave 2009. LNCS, vol. 6275, pp. 228-237. Springer, Heidelberg (2010)

17. De Paoli, F., Palmonari, M., Comerio, M., Maurino, A.: A meta-model for nonfunctional property descriptions of web services. In: Proceedings of the 2008 IEEE International Conference on Web Services, Washington, DC, USA, pp. 393-400. IEEE Computer Society, Los Alamitos (2008)

18. Paschke, A., Schnappinger-Gerull, E.: A categorization scheme for sla metrics. In: Service Oriented Electronic Commerce, pp. 25-40 (2006)

19. Ran, S.: A framework for discovering web services with desired quality of services attributes. In: ICWS, pp. 208-213 (2003)

20. Sahai, A., Durante, A., Machiraju, V.: Towards automated sla management for web services. Technical Report (2001)

21. Adel Serhani, M., Dssouli, R., Hafid, A., Sahraoui, H.: A qos broker based architecture for efficient web services selection. In: ICWS 2005: Proceedings of the IEEE International Conference on Web Services, Washington, DC, USA, pp. 113-120. IEEE Computer Society, Los Alamitos (2005)

22. Wang, Y., McIlraith, S.A., Yu, Y., Mylopoulos, J.: Monitoring and diagnosing software requirements. Autom. Softw. Eng. 16(1), 3-35 (2009)

23. Zadeh, L.A.: Fuzzy sets. Information and Control 8, 338-353 (1965)

24. Zeng, L., Benatallah, B., Ngu, A.H.H., Dumas, M., Kalagnanam, J., Chang, H.: Qos-aware middleware for web services composition. IEEE Trans. Softw. Eng. 30(5), 311-327 (2004) 